\title{
Multi-Objective Optimization of Manufacturing Lot Size Under Stochastic Demand
}

\author{
Maureen Nalubowa S. ${ }^{1,2,}$, Paul Kizito Mubiru' ${ }^{2}$ Jerry Ochola $^{3}$, Saul Namango ${ }^{4}$ \\ ${ }^{1,3,4}$ Moi University, Faculty of Engineering, Department of Manufacturing, Industrial and Textile Engineering, P.O. Box 3900- \\ 30100, Eldoret, Kenya. \\ 1,2Kyambogo University, Faculty of Engineering, Department of Mechanical and Production Engineering, P.O. Box 1, Kyambogo, \\ Uganda.
}

\begin{abstract}
In many manufacturing problems, multi-objective optimizations are representative models, as objectives are considered a conflict with one another. In real-life applications, optimizing a specific solution concerning one objective may end up in unacceptable results concerning the other objectives. Many Manufacturing companies operate under uncertainties and this affects the system performance. Stochastic product demand is one of the challenges faced by manufacturing companies and often affects the manufacturing system's performance and decision-making. Making the proper decisions regarding manufacturing lot-sizing problems is critical for any manufacturer because it makes the firm compete within the market. In this paper, Markov chains in conjunction with stochastic goal programming were used to develop an optimization model for the manufacturing lot size. The overachievement or under-achievement of the manufacturing lot size was determined by defining the goal constraints, deviation variables, priorities, and objective function. The different states of demand for the product with stochastic demand were represented by states of a Markov chain. Using the applied mathematics solver in MATLAB TM, the optimization model was then solved, determining the quantity of product to be manufactured in a given quarter of the year as demand changes from one state to another.
\end{abstract}

KEYWORDS: Multi-Objective, Manufacturing Lot Size, Optimization, Stochastic Product Demand, Stochastic Goal Programming

\section{INTRODUCTION}

Manufacturing companies experience a rapid and growing change where developments like customer orientation, globalization, and increasing market dynamics have led to a shift in both manufacturing and managerial principles which require more flexibility and effectiveness [1]. Many manufacturing companies operate under uncertainties [2] and this affects the system performance hence the ultimate decision on utilizing a production system at the initial stages [3]. Manufacturing companies are continuously trying to find efficiency to beat the challenges related to the market dynamics. Stochastic product demand is one of the important factors that affect the manufacturing system's performance. Practically, stochastic product demand is more realistic than other demand types, like constant or functions [4]. Understanding these uncertainties and their impacts (which can make it difficult to predict performance) when assessing the risk associated with a decision, are of major concern [5]. Having more orders, more different products, enlargement of factories, and increased number and size of workshops, have all led to more complications in production planning making the ordinary methods of optimization not able to resolve them [1]. Production planning is the pillar of any manufacturing operation, with the key purpose of determining the number of products to be manufactured considering the level of inventory to be shifted from one period to another to lessen both the overall costs of production and the inventory, meeting the customers' demand [6].

Making the proper decisions about the manufacturing lot size is incredibly important because it directly affects the system performance and productivity [7] and this is often key for any manufacturing firm that wishes to compete within the Market. Lot sizing problems have gotten an immediate effect on the system performance and productivity. Manufacturing Lot sizing is determining the amount of a given product that has to be manufactured in a specified period. Every production plan has got the main problem of determining the manufacturing lot size for every product. To own efficient production planning, lot allocation issues must be solved centered on the demand that has to be achieved and also the availability of inventory stock minimizing production costs by determining the optimal production quantity [8]. The smaller the manufacturing lot size, the less the holding cost but raises the ordering cost whereas the larger the manufacturing lot size, the more the holding cost but reducing the ordering cost. Based on 


\section{International Journal of Current Science Research and Review}

ISSN: 2581-8341

Volume 05 Issue 02 February 2022

DOI: 10.47191/ijcsrr/V5-i2-02, Impact Factor: 5.825

IJCSRR@ 2022

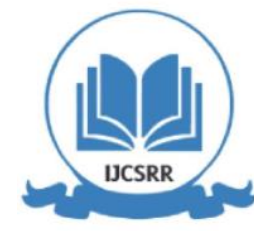

www.ijjcsrr.org

the concepts of lean production, it's preferable to have a smaller lot size because it prevents the buildup of inventory which comes with management and holding costs. The lot size recommended by a mathematical manufacturing lot size model would be the most effective because it accounts for the tradeoff between the costs involved [7]. Optimization is the process of finding (activity of selecting [9]) the simplest possible solution to a given problem by examining several alternatives (assessed after a predefined criterion) [10] and maybe done by adjusting the inputs to or characteristics of a device, mathematical process, or experiment to determine the minimum or maximum output [11]. The optimization problem contains three basic parameters that must be considered, that is, the objective function, a collection of variables, and a collection of constraints [12]. The objective of the optimization model depends on certain characteristics of the system, called variables or unknowns to determine the values of those variables that optimize the objective function, even though these variables are often restricted, or constrained in one way or the other. Brahimi et al. grouped optimization problems into four categories: process planning, layout design, re-configurability and planning, and, scheduling. In the beginning multi-objective optimization originally developed from areas including economic equilibrium and welfare theories, game theories, and pure mathematics. Consequently, many terms and fundamental ideas stem from these fields [13]. A realistic result to a multi-objective problem is to examine a collection of solutions, each satisfying the objectives at a satisfactory level without being controlled by another solution. Many, or maybe most, real engineering problems do have multiple objectives, that is, minimize cost, maximize performance, maximize reliability, and many others, of which are difficult but realistic problems [14]. The solution of multi-objective optimization (MOO) problems differs from single-objective optimization problems because there's no global optimal solution in an exceedingly mathematical sense, due to the contradictory nature of the set of objectives involved; that's, a result that minimizes all objectives at the same time doesn't exist [15]. Manufacturing companies must have the flexibility to regulate scalable production capacities and to respond rapidly to market demands making planning and scheduling complex in such a dynamic environment [16]. Markov chain is a powerful mathematical tool that's extensively accustomed to capturing the stochastic process of systems transitioning among different states [17]. Markov chains may be applied in modeling and performance evaluation as manufacturing systems show any unplanned behavior relating to breakdowns, unplanned time to process a component, and many others [18]. To tackle uncertainties in real-world manufacturing systems, goal programming and stochastic analysis must be put into the whole structure. Stochastic Goal Programming is a multi-criteria decision support model that provides "satisficing" solutions to a linear system under an uncertainty case from the normally expected utility viewpoint [19], [20].

Because many real-world optimization problems have got several inaccurate information estimates \& goals and conflicting criteria, the stochastic goal programming method suggests an analytical structure aid in modeling and solving such problems. Stochastic goal programming can cope with the inherent uncertainty and has been applied in several fields including Portfolio selection, project selection, resource allocation, Healthcare management, transportation, marketing [21], cash management [22], wealth management [23], economic development, energy consumption, workforce allocation, and greenhouse gas emissions [24], forest planning [25]. Little applications of stochastic goal programming in production planning in manufacturing systems are observed hence the necessity for multi-objective optimization of the manufacturing lot size under stochastic demand. This may be considered as a suggestion for production planners and practitioners accustomed to solving specific decision-making problems (optimal manufacturing lot size). Manufacturing companies will minimize overproduction when demand is low or underproduction when demand is high. As a result of fluctuations and uncertainties in demand, manufacturing companies are always challenged with determining optimal manufacturing lot sizes in production planning systems. Manufacturing companies are continuously searching for efficiency to beat the challenges related to the market dynamics. It's therefore important that these uncertain parameters be considered within the production planning process when developing a strong production plan because when neglected, production efficiency and system performance are affected [26]. Centering on external demands, manufacturing industries form their production plans having the principal goal of establishing the number (lot size) of products that can be produced for each period but meeting the demand and minimizing total costs [27]. In production planning, making the proper decisions about the lot size is extremely important because it directly affects the system performance and productivity [7] and this is often key for any manufacturing firm that desires to compete on market. As this is often complex moreover as important, it's been highly studied although, there's still a niche about showing the contributions to clarify the suitability of these methods used concerning each quite underlying manufacturing environment (regarding variations in demand and peaks of seasonality) [28]. Therefore this study aimed toward the 


\section{International Journal of Current Science Research and Review}

ISSN: 2581-8341

Volume 05 Issue 02 February 2022

DOI: 10.47191/ijcsrr/V5-i2-02, Impact Factor: 5.825

IJCSRR@ 2022

www.ijcsrr.org

development of a multi-objective optimization model for the manufacturing lot size under stochastic demand, establishing the overachievement or underachievement of the manufacturing lot size priorities desired.

\section{MATHEMATICAL MODEL FORMULATION}

A case of a manufacturing company whose products have got stochastic demand was considered. The demand for these products during each period over a finite fixed planning horizon was described as either favorable or unfavorable. The Markov chain approach ([29], [30], [31], [32], [1]) together with stochastic goal programming ([20], [33], [21], [34], [25], [22]) was adopted and also the states of a Markov chain represent possible states of demand for the finished products with the notations shown in Table 1.

Table 1: Key notations used in the Markov model

\begin{tabular}{c|l|c|l}
\hline$i, j$ & Set of states of demand & $M$ & Manufacturing lot-size \\
\hline$F$ & Favorable demand & $X_{i j}(p, q)$ & $\begin{array}{l}\text { Quantity of product p to be } \\
\text { manufactured in quarter q }\end{array}$ \\
\hline$U$ & Unfavorable demand & $N$ & Customer matrix \\
\hline$Q$ & Demand transition matrix & $C_{p}$ & Unit production cost \\
\hline$p$ & Product & $C_{h}$ & Unit holding cost \\
\hline$q$ & A quarter of the year & $C_{s}$ & Unit shortage cost \\
\hline$Z F, F U, U F, U U$ & State transitions & $D$ & Demand matrix \\
\hline$Z$ & Value of the objective function & $V$ & Inventory matrix \\
\hline$P_{k}$ & Preemptive priority of the $\mathrm{k}^{\text {th }}$ goal & $C$ & Production-Inventory cost matrix \\
\hline$d_{k}^{+}$ & Over achievement of the $\mathrm{k}^{\text {th }}$ goal & $B$ & Beginning Inventory \\
\hline$d_{k}^{-}$ & Under achievement of the $\mathrm{k}^{\text {th }}$ goal & $E$ & Ending Inventory \\
\hline
\end{tabular}

Average on-hand inventory, $V=(B+E) / 2$

Consider the customer matrix:

$$
N(p, q)=\left[\begin{array}{ll}
N_{F F}(p, q) & N_{F U}(p, q) \\
N_{U F}(p, q) & N_{U U}(p, q)
\end{array}\right]
$$

2.1 Demand transition probability

As demand changes from state $i$ to ievement state $j$ for $i, j \in\{F, U\}$, the associated demand transition probabilities are calculated as:

$$
Q_{i j}(p, q)=\frac{N_{i j}(p, q)}{N_{i f}(p, q)+N_{i u}(p, q)}
$$

This yields the demand transition matrix:

$$
Q(p, q)=\begin{array}{ccc}
\boldsymbol{F} & \boldsymbol{U} \\
\boldsymbol{U} & \left(\begin{array}{cc}
Q_{F F}(p, q) & Q_{F U}(p, q) \\
Q_{U F}(p, q) & Q_{U U}(p, q)
\end{array}\right)
\end{array}
$$

Then the demand matrix, the inventory matrix and the production-inventory cost matrix.

Demand matrix;

$$
D(A, 1)=\begin{array}{ccc}
\boldsymbol{F} & \boldsymbol{F} \\
\boldsymbol{U} & \left(\begin{array}{cc}
D_{F F}(A, 1) & D_{F U}(A, 1) \\
D_{U F}(A, 1) & D_{U U}(A, 1)
\end{array}\right)
\end{array}
$$

Inventory matrix;

$$
V(A, 1)=\begin{array}{ccc}
\boldsymbol{F} & \boldsymbol{F} & \boldsymbol{U} \\
\boldsymbol{U} & \left(\begin{array}{cc}
V_{F F}(A, 1) & V_{F U}(A, 1) \\
V_{U F}(A, 1) & V_{U U}(A, 1)
\end{array}\right)
\end{array}
$$

Production-inventory cost matrix; 


\section{International Journal of Current Science Research and Review}

ISSN: 2581-8341

Volume 05 Issue 02 February 2022

DOI: 10.47191/ijcsrr/V5-i2-02, Impact Factor: 5.825

When demand outweighs the amount produced then,

$$
C(p, q)=\left[\begin{array}{c}
C_{p} \\
+ \\
C_{h} \\
+ \\
C_{s}
\end{array}\right][D(p, q)-V(p, q)]
$$

Similarly, when the demand is less than the amount produced then,

$$
C(p, q)=C_{h}[V(p, q)-D(p, q)]
$$

Hence, as demand changes from state $i$ to state $j(i, j \in\{F, U\})$

$$
C(p, q)=\begin{array}{ccc}
\boldsymbol{F} & \boldsymbol{U} \\
\boldsymbol{U} & \left(\begin{array}{cc}
C_{F F}(p, q) & C_{F U}(p, q) \\
C_{U F}(p, q) & C_{U U}(p, q)
\end{array}\right)
\end{array}
$$

Where $C(p, q)=$ production-inventory cost matrix.

\subsection{Expected demand, inventory, production-inventory costs, and manufacturing lot-size}

Expected demand

$$
\begin{aligned}
& \text { Favorable Demand } E\left[D_{F}(p, q)\right]=Q_{F F}(p, q) D_{F F}(p, q)+Q_{F U}(p, q) D_{F U}(p, q) \\
& \text { Unfavorable Demand } E\left[D_{U}(p, q)\right]=Q_{U F}(p, q) D_{U F}(p, q)+Q_{U U}(p, q) D_{U U}(p, q)
\end{aligned}
$$

Expected inventory

$$
\begin{aligned}
& \text { Favorable Demand } E\left[V_{F}(p, q)\right]=Q_{F F}(p, q) V_{F F}(p, q)+Q_{F U}(p, q) V_{F U}(p, q) \\
& \text { Unfavorable Demand } E\left[V_{U}(p, q)\right]=Q_{F U}(p, q) V_{U F}(p, q)+Q_{U U}(p, q) V_{U U}(p, q)
\end{aligned}
$$

Expected production-inventory costs

$$
\begin{aligned}
& \text { Favorable Demand } E\left[C_{F}(p, q)\right]=Q_{F F}(p, q) C_{F F}(p, q)+Q_{F U}(p, q) C_{F U}(p, q) \\
& \text { Unfavorable Demand } E\left[C_{U}(p, q)\right]=Q_{U F}(p, q) C_{U F}(p, q)+Q_{U U}(p, q) C_{U U}(p, q)
\end{aligned}
$$

Expected manufacturing lot-size

Favorable demand

$$
E\left[M_{F}(p, q)\right]=\left\{\begin{array}{cc}
E\left[D_{F}(p, q)\right]-E\left[V_{F}(p, q)\right] & \text { if } E\left[D_{F}(p, q)\right]>E\left[V_{F}(p, q)\right] \\
0 & \text { otherwise }
\end{array}\right.
$$

Unfavorable demand

$$
E\left[M_{U}(p, q)\right]=\left\{\begin{array}{cc}
E\left[D_{U}(p, q)\right]-E\left[V_{U}(p, q)\right] & \text { if } E\left[D_{U}(p, q)\right]>E\left[V_{U}(p, q)\right] \\
0 & \text { otherwise }
\end{array}\right.
$$

\subsection{Stochastic goal programming formulation}

The stochastic goal programming model was formulated by setting priorities, defining the objective function, and formulating the goal constraints as follows:

Set priorities

$\mathrm{P}_{1}$ : Produce a batch of $E\left[M_{F}(p, q)\right]$ units when demand is favorable

$\mathrm{P}_{2}$ : Produce a batch of $E\left[M_{U}(p, q)\right]$ units when demand is unfavorable

$\mathrm{P}_{3}$ : Total production-inventory cost must not exceed $E\left[C_{F}(p, q)\right]$ when demand is favorable

$\mathrm{P}_{4}$ : Total production-inventory cost must not exceed $E\left[C_{U}(p, q)\right]$ when demand is unfavorable 


\section{International Journal of Current Science Research and Review}

ISSN: 2581-8341

Volume 05 Issue 02 February 2022

DOI: 10.47191/ijcsrr/V5-i2-02, Impact Factor: 5.825

IJCSRR@ 2022

www.ijcsrr.org

Objective function

$$
\text { Minimise } Z=\sum_{k=1}^{4} \quad \sum_{p=1}^{3} \quad \sum_{q=1}^{3} P_{k}(p, q)\left[d_{k}^{+}+d_{k}^{-}\right]
$$

Goal constraints

P1: Manufacturing lot-size $E\left[M_{F}(p, q)\right]$ - favorable demand

$X_{F F}(p, q)+X_{F U}(p, q)+d_{1}^{-}-d_{1}^{+}=E\left[M_{F}(p, q)\right]$

P2: Manufacturing lot-size $E\left[M_{U}(p, q)\right]$ - unfavorable demand

$X_{U F}(p, q)+X_{U U}(p, q)+d_{2}^{-}-d_{2}^{+}=E\left[M_{U}(p, q)\right]$

P3: Total production-inventory cost - favorable demand

$C_{F F}(p, q) X_{F F}(p, q)+C_{F U}(p, q) X_{F U}(p, q)-d_{3}^{+}=E\left[C_{F}(p, q)\right]$

P4: Total production-inventory cost - unfavorable demand

$C_{U F}(p, q) X_{U F}(p, q)+C_{U U}(p, q) X_{U U}(p, q)-d_{4}^{+}=E\left[C_{U}(p, q)\right]$

2.4 Stochastic goal programming model for manufacturing lot-size

Minimise $Z=\sum_{k=1}^{4} \quad \sum_{p=1}^{3} \quad \sum_{q=1}^{3} P_{k}(p, q)\left[d_{k}^{+}+d_{k}^{-}\right]$

Subject to:

$$
\begin{aligned}
& X_{F F}(p, q)+X_{F U}(p, q)+d_{1}^{-}-d_{1}^{+}=E\left[M_{F}(p, q)\right] \\
& X_{U F}(p, q)+X_{U U}(p, q)+d_{2}^{-}-d_{2}^{+}=E\left[M_{U}(p, q)\right] \\
& C_{F F}(p, q) X_{F F}(p, q)+C_{F U}(p, q) X_{F U}(p, q)-d_{3}^{+}=E\left[C_{F}(p, q)\right] \\
& C_{U F}(p, q) X_{U F}(p, q)+C_{U U}(p, q) X_{U U}(p, q)-d_{4}^{+}=E\left[C_{U}(p, q)\right] \\
& X_{F F}(p, q), X_{F U}(p, q), X_{U F}(p, q), X_{U U}(p, q), d_{1}^{-}, d_{1}^{+}, d_{2}^{-}, d_{2}^{+}, d_{3}^{+}, d_{4}^{+} \geq 0
\end{aligned}
$$

\section{CASE STUDY}

In this section, a real case application from Movit Products Uganda limited was used to demonstrate the applicability of the proposed mathematical models. The manufacturing industry manufactures, distributes, and sells skin care, hair $\&$ nail care products. The

\begin{tabular}{|c|c|c|c|c|c|}
\hline Month & Week & $\begin{array}{l}\text { Customers } \\
(\mathrm{N})\end{array}$ & $\begin{array}{l}\text { Demand } \\
\left(\mathbf{x} 10^{3}\right)\end{array}$ & $\begin{array}{l}\text { On hand inventory }(\mathrm{V}) \\
\left(\mathbf{x 1 0}^{3}\right)\end{array}$ & $\begin{array}{l}\text { State of } \\
\text { demand }(i)\end{array}$ \\
\hline \multirow{4}{*}{1} & 1 & 15 & 308 & 5263 & $\mathrm{U}$ \\
\hline & 2 & 29 & 2891 & 7337 & $\mathrm{~F}$ \\
\hline & 3 & 24 & 1757 & 7081 & $\mathrm{U}$ \\
\hline & 4 & 38 & 6619 & 5654 & F \\
\hline \multirow{4}{*}{2} & 1 & 8 & 231 & 3525 & $\mathrm{U}$ \\
\hline & 2 & 17 & 2046 & 6243 & $\mathrm{U}$ \\
\hline & 3 & 15 & 1617 & 5922 & $\mathrm{U}$ \\
\hline & 4 & 45 & 4443 & 5951 & $\mathrm{~F}$ \\
\hline \multirow{4}{*}{3} & 1 & 14 & 559 & 3765 & $\mathrm{U}$ \\
\hline & 2 & 37 & 3686 & 4738 & $\mathrm{~F}$ \\
\hline & 3 & 28 & 1537 & 4980 & F \\
\hline & 4 & 44 & 5626 & 5746 & F \\
\hline
\end{tabular}
numerical illustration contains real data for the first quarter of the year, which was collected and then reduced to usable dimensions as shown in Table 2. Data classification by state of demand was made, analyzed, and used in the proposed mathematical model.

Considering a product $\mathrm{D}$, for a given week, demand is favorable (state $\mathrm{F}$ ) if $\mathrm{N}_{\mathrm{ij}}>26$ otherwise demand is unfavorable (state $\mathrm{U}$ ) if $\mathrm{N}_{\mathrm{ij}} \leq 26$ as shown in Table 2 .

Table 2: Data classification by state of demand for product D 


\section{International Journal of Current Science Research and Review}

ISSN: 2581-8341

Volume 05 Issue 02 February 2022

DOI: 10.47191/ijcsrr/V5-i2-02, Impact Factor: 5.825

IJCSRR@ 2022

www.ijcsrr.org

Tables $3 \mathrm{a}, 3 \mathrm{~b}$, and $3 \mathrm{c}$ show the overstocking or understocking of product $\mathrm{D}$ with the corresponding holding or shortage costs in the first quarter of the year.

Table 3a: Overstocking and understocking with holding and shortage costs for month 1

\begin{tabular}{lllll}
\hline Week & Demand $(\mathbf{D}) \mathbf{( \mathbf { x 1 0 } ^ { \mathbf { 3 } } )}$ & $\begin{array}{l}\text { On hand inventory }(\mathbf{V}) \\
\left(\mathbf{x 1 0}^{\mathbf{3}}\right)\end{array}$ & over/under stocking & $\begin{array}{l}\text { Holding/shortage } \\
\text { costs }\end{array}$ \\
\hline 1 & 308 & 5263 & 4955 & 3121.65 \\
2 & 2891 & 7337 & 4446 & 2800.98 \\
3 & 7081 & 5324 & 3354.12 \\
4 & 1757 & 5654 & -965 & 3343.725 \\
\hline
\end{tabular}

Table 3b: Overstocking and understocking with holding and shortage costs for month 2

\begin{tabular}{|c|c|c|c|c|}
\hline Week & Demand (D) $\left(\times 10^{3}\right)$ & $\begin{array}{l}\text { On hand inventory }(\mathrm{V}) \\
\left(\times 10^{3}\right)\end{array}$ & over/under stocking & $\begin{array}{l}\text { Holding/shortage } \\
\text { costs }\end{array}$ \\
\hline 1 & 231 & 3525 & 3294 & 2075.22 \\
\hline 2 & 2046 & 6243 & 4197 & 2644.11 \\
\hline 3 & 1617 & 5922 & 4305 & 2712.15 \\
\hline 4 & 4443 & 5951 & 1508 & 950.04 \\
\hline
\end{tabular}

Table 3c: Overstocking and understocking with holding and shortage costs for month 3

\begin{tabular}{lllll}
\hline Week & Demand $(\mathbf{D})\left(\mathbf{x 1 0}^{\mathbf{3}}\right)$ & $\begin{array}{l}\text { On hand inventory }(\mathbf{V}) \\
\left(\mathbf{x 1 0}^{\mathbf{3}}\right)\end{array}$ & over/under stocking & $\begin{array}{l}\text { Holding/shortage } \\
\text { costs }\end{array}$ \\
\hline 1 & 559 & 3765 & 3206 & 2019.78 \\
2 & 3686 & 4738 & 1052 & 662.76 \\
3 & 4980 & 3443 & 2169.09 \\
4 & 1537 & 5746 & 120 & 75.6 \\
\hline
\end{tabular}

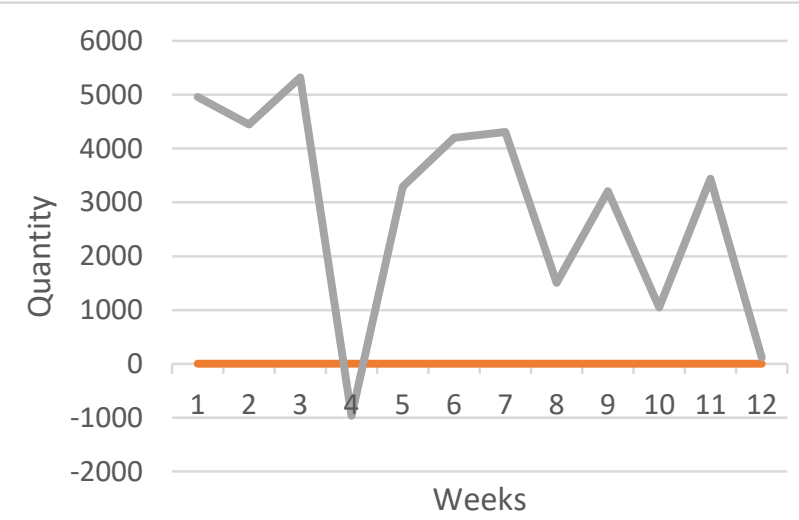

Figure 1: Overstocking and understocking of product D

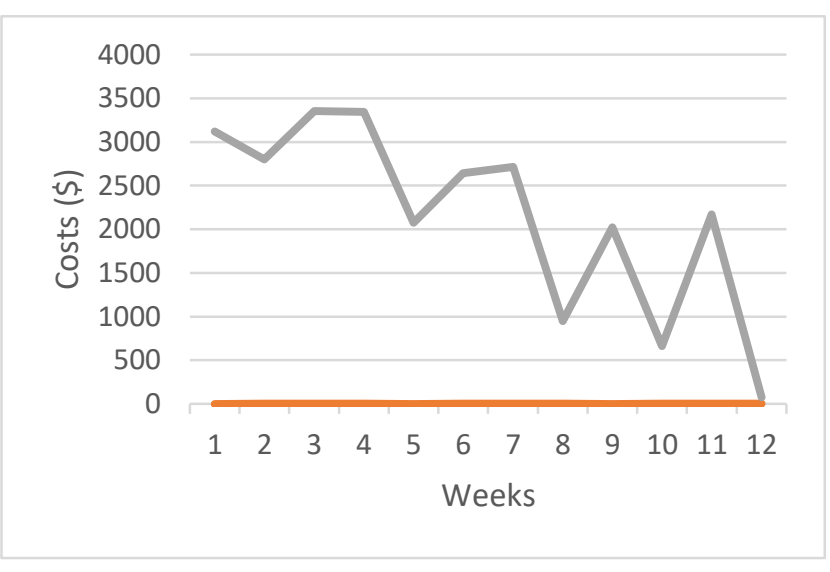

Figure 2: Holding and Shortage costs

3.1 State transitions and on-hand inventory

For a particular state transition, given the beginning and ending inventory, the average on-hand inventory was calculated as presented in Table 4. 


\section{International Journal of Current Science Research and Review}

ISSN: 2581-8341

Volume 05 Issue 02 February 2022

DOI: 10.47191/ijesrr/V5-i2-02, Impact Factor: 5.825

IJCSRR@ 2022

www.ijcsrr.org

Table 4: Average on-hand inventory for product D

\begin{tabular}{llll}
\hline $\begin{array}{c}\text { State transitions } \\
(\boldsymbol{i}, \boldsymbol{j})\end{array}$ & Beginning inventory $(\mathbf{B})$ & Ending inventory $(\mathbf{E})$ & $\begin{array}{c}\text { Average on-hand inventory } \\
\boldsymbol{V}=(\boldsymbol{B}+\boldsymbol{E}) / \mathbf{2}\end{array}$ \\
\hline FF & & & 5363 \\
FU & 4980 & 5746 & 5423 \\
UF & 7081 & 3765 & 6037.5 \\
UU & 7337 & 4738 & 6082.5 \\
\hline
\end{tabular}

From Equation (1) section 2, the average on-hand inventory was calculated giving;

$$
V_{F F}(D, 1)=5363 \quad V_{F U}(D, 1)=5423 \quad V_{U F}(D, 1)=6037.5 \quad V_{U U}(D, 1)=6082.5
$$

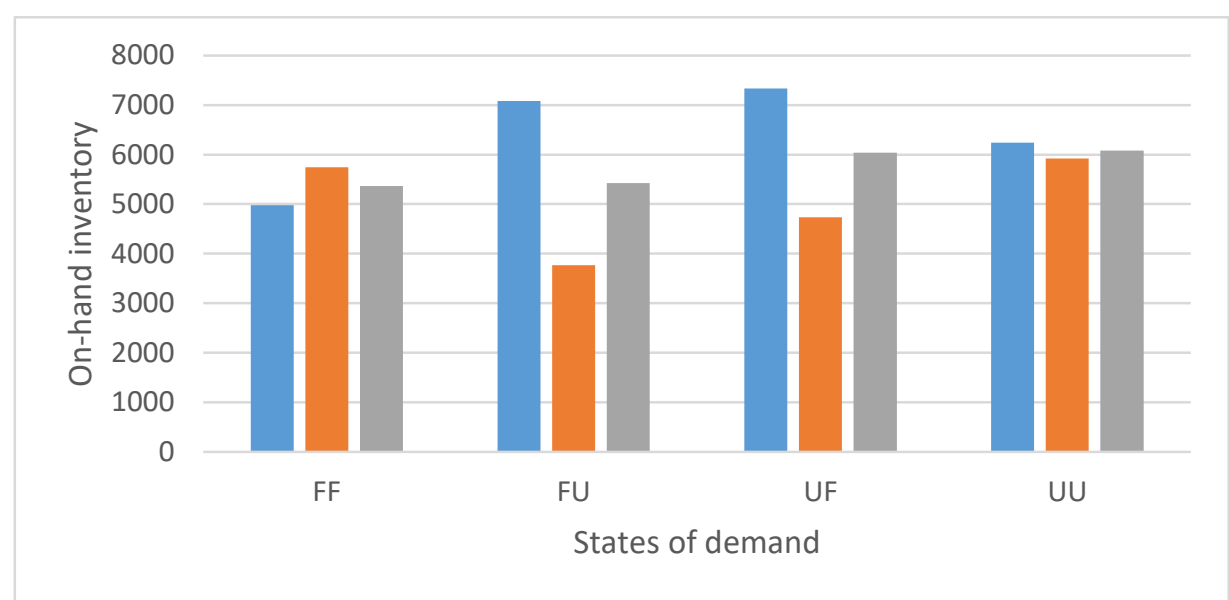

Figure 3: Average on-hand inventory and state transitions

\subsection{Demand transition probabilities}

Data classification by state transition was done as illustrated in Table 5 and then used to calculate the demand transition probabilities for the product.

Table 5: Data classification by state-transition for product D

\begin{tabular}{|c|c|c|c|}
\hline Month & $\begin{array}{c}\text { State transition } \\
(\boldsymbol{i}, \boldsymbol{j})\end{array}$ & $\begin{array}{l}\text { Number of customers } \\
\qquad N_{i j}(A, 1)\end{array}$ & $\begin{array}{l}\text { Demand } \\
D_{i j}(A, 1)\end{array}$ \\
\hline \multirow{4}{*}{1} & $\mathrm{FF}$ & 0 & 0 \\
\hline & FU & 53 & 4648 \\
\hline & UF & 106 & 11575 \\
\hline & UU & 0 & 0 \\
\hline \multirow{4}{*}{2} & $\mathrm{FF}$ & 0 & 0 \\
\hline & FU & 0 & 0 \\
\hline & UF & 60 & 6060 \\
\hline & UU & 57 & 5940 \\
\hline \multirow{4}{*}{3} & FF & 137 & 12386 \\
\hline & FU & 0 & 0 \\
\hline & UF & 51 & 4245 \\
\hline & UU & 0 & 0 \\
\hline
\end{tabular}




\section{International Journal of Current Science Research and Review}

ISSN: 2581-8341

Volume 05 Issue 02 February 2022

DOI: 10.47191/ijcsrr/V5-i2-02, Impact Factor: 5.825

IJCSRR@ 2022

From table 5, the Totals for customers and demand as it changes from one state to another are;

Customers: $N_{F F}(D, 1)=137$ $N_{F U}(D, 1)=53$

$N_{U F}(D, 1)=106+60+51=217$

$N_{U U}(D, 1)=57$

Demand: $D_{F F}(D, 1)=12386$

$$
D_{F U}(D, 1)=4648
$$

$$
D_{U F}(D, 1)=11575+6060+4245=21880 \quad D_{U U}(D, 1)=5940
$$

From Equation (3) in section 2, the demand transition probabilities are;

$$
\begin{aligned}
& Q_{F F}(D, 1)=\frac{N_{F F}(D, 1)}{N_{F F}(D, 1)+N_{F U}(D, 1)}=\frac{137}{137+53}=0.7211 \\
& Q_{F U}(D, 1)=\frac{N_{F U}(D, 1)}{N_{F F}(D, 1)+N_{F U}(C, 1)}=\frac{53}{137+53}=0.2789 \\
& Q_{U F}(D, 1)=\frac{N_{U F}(D, 1)}{N_{U F}(D, 1)+N_{U U}(D, 1)}=\frac{217}{217+57}=0.7920 \\
& Q_{U U}(D, 1)=\frac{N_{U U}(D, 1)}{N_{U F}(D, 1)+N_{U U}(D, 1)}=\frac{57}{217+57}=0.2080
\end{aligned}
$$

Hence the demand transition matrix as from equation (4),

$$
Q(D, 1)=\begin{array}{ccc}
\boldsymbol{F} & \boldsymbol{F} & \boldsymbol{U} \\
\boldsymbol{U} & \left(\begin{array}{ll}
0.7211 & 0.2789 \\
0.7920 & 0.2080
\end{array}\right)
\end{array}
$$

\subsection{Demand matrix, inventory matrix, and production-inventory cost matrix}

The demand matrix, the inventory matrix, and the production-inventory cost matrix were developed as follows.

From Equation (5), the demand matrix becomes;

$$
D(D, 1)=\begin{array}{ccc}
\boldsymbol{F} & \boldsymbol{F} & \boldsymbol{U} \\
\boldsymbol{U} & \left(\begin{array}{ll}
12386 & 4648 \\
21880 & 5940
\end{array}\right)
\end{array}
$$

From Equation (6), the Inventory matrix becomes;

$$
\left.V(D, 1)=\begin{array}{l}
F \\
U
\end{array} \quad \begin{array}{cc}
F 363 & 5423 \\
6037.5 & 6082.5
\end{array}\right)
$$

Production-inventory cost matrix

The production-inventory cost matrix is then computed for the product From Equations (7), (8), and (9).

$$
\begin{aligned}
& \text { Unit production cost, } C_{p}(D)=\$ 31.5 \\
& \text { Unit holding cost, } C_{h}(D)=\$ 0.63 \\
& \text { Unit shortage cost, } C_{s}(D)=\$ 3.465 \\
& C_{F F}(D, 1)=\left(C_{p}(D)+C_{h}(D)+C_{s}(D)\right)\left(D_{F F}(D, 1)-V_{F F}(D, 1)\right) \\
& C_{F F}(D, 1)=(31.5+0.63+3.465)(12386-5363)=249983.685 \\
& C_{F U}(D, 1)=C_{h}(D)\left(V_{F U}(D, 1)-D_{F U}(D, 1)\right) \\
& C_{F U}(D, 1)=0.63(5423-4648)=488.25 \\
& C_{U F}(D, 1)=\left(C_{p}(D)+C_{h}(D)+C_{s}(D)\right)\left(D_{U F}(D, 1)-V_{U F}(D, 1)\right) \\
& C_{U F}(D, 1)=(31.5+0.63+3.465)(21880-6037.5)=563913.7875 \\
& C_{U U}(D, 1)=C_{h}(D)\left(V_{U U}(D, 1)-D_{U U}(D, 1)\right) \\
& C_{U U}(D, 1)=(0.63)(6082.5-5940)=89.775
\end{aligned}
$$

Hence,

$$
\begin{array}{cccc}
C(D, 1)= & \boldsymbol{F} & \boldsymbol{F} & \boldsymbol{U} \\
& \boldsymbol{U} & \left(\begin{array}{cc}
C_{F F}(D, 1) & C_{F U}(D, 1) \\
C_{U F}(D, 1) & C_{U U}(D, 1)
\end{array}\right) \\
C(D, 1)= & \boldsymbol{F} & \boldsymbol{F} & \boldsymbol{U} \\
& \boldsymbol{U} & \left(\begin{array}{ccc}
249983.685 & 488.25 \\
563913.7875 & 89.775
\end{array}\right)
\end{array}
$$




\section{International Journal of Current Science Research and Review}

ISSN: 2581-8341

Volume 05 Issue 02 February 2022

DOI: 10.47191/ijcsrr/V5-i2-02, Impact Factor: 5.825

IJCSRR@ 2022

www.ijjcsrr.org

\subsection{Expected demand, inventory, production-inventory costs, and manufacturing lot-size}

Expected demand

After generating the demand transition matrix and formulating the production-inventory cost matrix, the expected demand expected inventory, and expected production-inventory costs are computed for the product considering both favorable and unfavorable demand as shown below;

Favorable demand (F) was computed from equation (10)

$$
\begin{aligned}
& E\left[D_{F}(D, 1)\right]=Q_{F F}(D, 1) * D_{F F}(D, 1)+Q_{F U}(D, 1) * D_{F U}(D, 1) \\
& E\left[D_{F}(D, 1)\right]=(0.7211 * 12386)+(0.2789 * 4648) \\
& E\left[D_{F}(D, 1)\right]=11227.8718 \text { units }
\end{aligned}
$$

Unfavorable demand (U) was computed from equation (11)

$$
\begin{aligned}
& E\left[D_{U}(D, 1)\right]=Q_{U F}(D, 1) * D_{U F}(D, 1)+Q_{U U}(D, 1) * D_{U U}(D, 1) \\
& E\left[D_{U}(D, 1)\right]=(0.7920 * 21880)+(0.2080 * 5940) \\
& E\left[D_{U}(D, 1)\right]=18564.48 \text { units }
\end{aligned}
$$

Computation of the expected inventory considering both favorable and unfavorable demand for the product was computed from equation (12) as follows:

Favorable demand (F)

$$
\begin{aligned}
& E\left[V_{F}(D, 1)\right]=Q_{F F}(D, 1) * V_{F F}(D, 1)+Q_{F U}(D, 1) * V_{F U}(D, 1) \\
& E\left[V_{F}(D, 1)\right]=(0.7211 * 5363)+(0.2789 * 5423) \\
& E\left[V_{F}(D, 1)\right]=5379.734 \text { units }
\end{aligned}
$$

Unfavorable demand (U) was computed from equation (13) as follows

$$
\begin{aligned}
& E\left[V_{U}(D, 1)\right]=Q_{U F}(D, 1) * V_{U F}(D, 1)+Q_{U U}(D, 1) * V_{U U}(D, 1) \\
& E\left[V_{U}(D, 1)\right]=(0.7920 * 6037.5)+(0.2080 * 6082.5) \\
& E\left[V_{U}(D, 1)\right]=6046.86 \text { units }
\end{aligned}
$$

Expected production-Inventory costs

The expected production-Inventory costs are then computed for the product considering both favorable and unfavorable demand results were computed from equations (14) and (15) as follows;

Favorable demand (F)

$$
\begin{aligned}
& E\left[C_{F}(D, 1)\right]=Q_{F F}(D, 1) * C_{F F}(D, 1)+Q_{F U}(D, 1) * C_{F U}(D, 1) \\
& E\left[C_{F}(D, 1)\right]=(0.7211 * 249983.685)+(0.2789 * 488.25) \\
& E\left[C_{F}(D, 1)\right]=\$ 180399.4082
\end{aligned}
$$

Unfavorable demand (U)

$$
\begin{aligned}
& E\left[C_{U}(D, 1)\right]=Q_{U F}(D, 1) * C_{U F}(D, 1)+Q_{U U}(D, 1) * C_{U U}(D, 1) \\
& E\left[C_{U}(D, 1)\right]=(0.7920 * 563913.7875)+(0.2080 * 89.775) \\
& E\left[C_{U}(D, 1)\right]=\$ 446638.3929
\end{aligned}
$$

Expected manufacturing lot size

Computation of the expected manufacturing lot size considering both favorable and unfavorable demand for the product yields was computed from equations (16) and (17) as follows:

Favorable demand (F)

$$
\begin{gathered}
E\left[M_{F}(D, 1)\right]=\left(\begin{array}{c}
E\left[D_{F}(D, 1)\right]-E\left[V_{F}(D, 1)\right] \text { if } E\left[D_{F}(D, 1)\right]>E\left[V_{F}(D, 1)\right] \\
0 \\
\text { otherwise }
\end{array}\right) \\
E\left[M_{F}(D, 1)\right]=E\left[D_{F}(D, 1)\right]-E\left[V_{F}(D, 1)\right] \\
E\left[M_{F}(D, 1)\right]=11227.8718-5379.734=5848.1378 \text { units }
\end{gathered}
$$




\section{International Journal of Current Science Research and Review}

ISSN: 2581-8341

Volume 05 Issue 02 February 2022

DOI: 10.47191/ijcsrr/V5-i2-02, Impact Factor: 5.825

IJCSRR @ 2022

www.ijesrr.org

Unfavorable demand (U)

$$
\begin{gathered}
E\left[M_{U}(D, 1)\right]=\left(\begin{array}{c}
E\left[D_{U}(D, 1)\right]-E\left[V_{U}(D, 1)\right] \text { if } E\left[D_{U}(D, 1)\right]>E\left[V_{U}(D, 1)\right] \\
0 \\
\text { otherwise }
\end{array}\right) \\
E\left[M_{U}(D, 1)\right]=E\left[D_{U}(D, 1)\right]-E\left[V_{U}(D, 1)\right] \\
E\left[M_{U}(D, 1)\right]=18564.48-6046.86=12517.62 \text { units }
\end{gathered}
$$

\subsection{Stochastic goal programming model}

The stochastic goal programming model for the product was formulated by setting priorities, defining the objective function, and formulating the goal constraints as follows:

Priorities set

$P_{1}$ : Produce a batch of 5848.1378 units when demand is initially favorable

$P_{2}$ : Produce a batch of 12517.62 units when demand is initially unfavorable

$P_{3}$ :Total production_inventory costs must not exceed $\$ 180399.4082$

when demand is favorable $P_{4}$ : Total production_inventory costs must not exceed $\$ 446638.3929$

when demand is unfavorable

Objective function

$$
\text { Minimize } Z=\sum_{k=1}^{4}\left[P_{K}(D, 1) d_{k}^{+}+P_{K}(D, 1) d_{k}^{-}\right]
$$

Goal constraints

Manufacturing lot size

$$
\begin{aligned}
& X_{F F}(D, 1)+X_{F U}(D, 1)+d_{1}^{-}=5848.1378 \text { (Favorable demand) } \\
& X_{U F}(D, 1)+X_{U U}(D, 1)+d_{2}^{-}=12517.62 \text { (Unfavorable demand) }
\end{aligned}
$$

Total production-Inventory costs

249983.685 $X_{F F}(D, 1)+488.25 X_{F U}(D, 1)-d_{3}^{+}=180399.4082$ (Favorable demand)

$563913.7875 X_{U F}(D, 1)+89.775 X_{U U}(D, 1)-d_{4}^{+}=446638.3929$ (Unfavorable demand)

Non-negativity

$$
X_{F F}(D, 1), X_{F U}(D, 1), X_{U F}(D, 1), X_{U U}(D, 1), d_{1}^{-}, d_{2}^{-}, d_{3}^{+}, d_{4}^{+} \geq 0
$$

\subsection{Stochastic goal programming model for manufacturing lot size}

The stochastic goal programming model for manufacturing lot size was then developed for the product as below. This determines the quantity of the product to manufacture in the first quarter of the year when demand changes from state $i$ to state $j$ for $i, j \in\{F, U\}$, establishing the over-achievement or under achievement of the manufacturing lot size priorities desired.

Minimize $Z=\sum_{k=1}^{4}\left[P_{K}(D, 1) d_{k}^{+}+P_{K}(D, 1) d_{k}^{-}\right]$

Subject to:

$$
\begin{aligned}
& X_{F F}(D, 1)+X_{F U}(D, 1)+d_{1}^{-}=5848.1378 \\
& X_{U F}(D, 1)+X_{U U}(D, 1)+d_{2}^{-}=12517.62 \\
& 249983.685 X_{F F}(D, 1)+488.25 X_{F U}(D, 1)-d_{3}^{+}=180399.4082 \\
& 563913.7875 X_{U F}(D, 1)+89.775 X_{U U}(D, 1)-d_{4}^{+}=446638.3929 \\
& X_{F F}(D, 1), X_{F U}(D, 1), X_{U F}(D, 1), X_{U U}(D, 1), d_{1}^{-}, d_{2}^{-}, d_{3}^{+}, d_{4}^{+} \geq 0
\end{aligned}
$$

Where:

$d_{1}^{-}, d_{2}^{-}=$slack variables

$d_{3}^{+}, d_{4}^{+}=$surplus variables

$X_{F F}(D, 1)$ - manufacturing lot size of product $\mathrm{D}$ when initially favorable demand remains favorable

$X_{F U}(D, 1)$ - manufacturing lot size of product $\mathrm{D}$ when initially favorable demand becomes unfavorable

$\mathrm{X}_{\mathrm{UF}}(\mathrm{D}, 1)$ - manufacturing lot size of product $\mathrm{D}$ when initially unfavorable demand becomes favorable

$X_{U U}(D, 1)$ - manufacturing lot size of product $\mathrm{D}$ when initially unfavorable demand remains unfavorable 


\section{International Journal of Current Science Research and Review}

ISSN: 2581-8341

Volume 05 Issue 02 February 2022

DOI: 10.47191/ijcsrr/V5-i2-02, Impact Factor: 5.825

IJCSRR@ 2022

www.ijcsrr.org

\section{RESULTS AND DISCUSSIONS}

In this study, the stochastic goal programming model for the product was solved using MATLAB. The values were inserted in MATLAB TM ([35], [36]) and using the linprog solver, an optimal solution was obtained with the values as shown in Table 6:

Table 6: Optimal solution from MATLAB

\begin{tabular}{lllllllll}
\hline Variables & $\mathrm{X}_{\mathrm{FF}}(\mathrm{D}, 1)$ & $\mathrm{X}_{\mathrm{FU}}(\mathrm{D}, 1)$ & $\mathrm{X}_{\mathrm{UF}}(\mathrm{D}, 1)$ & $\mathrm{X}_{\mathrm{UU}}(\mathrm{D}, 1)$ & $\mathrm{d}_{1}{ }^{-}$ & $\mathrm{d}_{2}{ }^{-}$ & $\mathrm{d}_{3}{ }^{+}$ & $\mathrm{d}_{4}{ }^{+}$ \\
\hline values & 0 & 369.4816 & 0 & 12518 & 5478.7 & 0 & 0 & 677130 \\
\hline
\end{tabular}

The results highlight the optimal values of the manufacturing lot size of product A in the first quarter of the year as demand changes from one state to another. The results were analyzed and discussed based on the priorities set and the optimal values achieved as seen from table 6 .

The improvement of the solution from the case is establishing the over-achievement and under achievement of the manufacturing lot size priorities desired during production planning. An expansion, in this case, is incorporated in Markov chains which considers changes from one state to another. As seen from table 6, for cases where initially demand is favorable and unfavorable, more products shouldn't be manufactured but use what is already in stock as it is enough to meet the demand since the model predicts 0 manufacturing lot size of product $\mathrm{A}$ in the first quarter of the year.

The model also predicts the manufacturing lot size of product A of 2.3729units and 104.0840 units when initially favorable demand becomes unfavorable and unfavorable demand remains unfavorable respectively. Meaning these number of products should be produced to meet demand.

Table 7: Expected goal values and actual stochastic solution with over and under achievement

\begin{tabular}{lccccc}
\hline $\begin{array}{l}\text { Goals/ } \\
\text { priorities }\end{array}$ & $\begin{array}{l}\text { Expected value } \\
\text { from Goal }\end{array}$ & $\begin{array}{l}\text { Value of the } \\
\text { stochastic solution }\end{array}$ & Deviation & Over-achievement & Under-achievement \\
\hline 1 & 5848.1378 & 5848.1816 & 0.0438 & & 5478.7 \\
\hline 2 & 12517.62 & 12518 & 0.38 & & 0 \\
\hline 3 & 180399.4082 & 180399.3912 & 0.017 & 0 & \\
\hline 4 & 446638.3929 & 446673.45 & 35.0571 & 677130 & \\
\hline
\end{tabular}

With the set priorities and expected values from each goal, the results from table 7 show the importance of utilizing the available sources of information when generating a plan.

As observed from table 7, Priorities 1, 2, and 3 can be fully achieved however, an underachievement of 5478.7 units is realized in the first quarter when demand is initially favorable (state F).

Priority 4 is partially achieved as the actual stochastic solution is slightly higher than the expected goal value targeted productioninventory costs in the first quarter when demand is initially unfavorable (state $U$ ) and an over-achievement of 677130 units is realized.

\section{CONCLUSION}

A stochastic goal programming model that optimizes the manufacturing lot size under demand uncertainty was presented in this paper. The model determines the quantity of the product (with demand uncertainty to be produced in the first quarter of the year when demand changes from state $i$ to state $j$ for $i, j \in\{F, U\}$, establishing the over-achievement or underachievement of the manufacturing lot size priorities desired. The decision of whether or not to produce more units is modeled using Markov chains in conjunction with stochastic goal programming. The model was solved with the help of MATLAB software environment and the results indicate the optimal manufacturing lot sizes as demand changes from one state to another, establishing the over-achievement or underachievement of the manufacturing lot size priorities desired.

Further research is sought to extend the proposed model to handle multiple products under demand and price uncertainty. In addition, weighted goal programming can be introduced to improve computational efficiency while handling pre-emptive priorities of the product. 


\section{International Journal of Current Science Research and Review}

ISSN: 2581-8341

Volume 05 Issue 02 February 2022

DOI: 10.47191/ijesrr/V5-i2-02, Impact Factor: 5.825

IJCSRR @ 2022

www.ijcsrr.org

\section{ACKNOWLEDGMENT}

The author would like to thank the management and staff of Movit Products Uganda Limited for the support extended during the data collection exercise.

\section{REFERENCES}

1. C. Tochukwu and I. Hyacinth, "Agent Based Markov Chain for Job Shop Scheduling and Control: Review of the Modeling Technigue," 2015. [Online]. Available: www.ijiset.com.

2. K. P. Mubiru, “458 An EOQ Model For Multi-Item Inventory With Stochastic Demand,” Int. J. Eng. Res. Technol., vol. 2, no. 7, pp. 2485-2492, 2013.

3. A. Vafadar, M. Tolouei-Rad, and K. Hayward, "Evaluation of the Effect of Product Demand Uncertainty on Manufacturing System Selection,” Procedia Manuf., vol. 11, no. June, pp. 1735-1743, 2017, doi: 10.1016/j.promfg.2017.07.301.

4. A. K. Dhaiban and N. Aziz, "Stochastic demand of production-inventory system with shortage," AIP Conf. Proc., vol. 2138, no. March, 2019, doi: 10.1063/1.5121034.

5. M. Assid, A. Gharbi, and A. Hajji, "Production planning of an unreliable hybrid manufacturing-remanufacturing system under uncertainties and supply constraints," Comput. Ind. Eng., vol. 136, no. October 2018, pp. 31-45, 2019, doi: 10.1016/j.cie.2019.06.061.

6. O. Olanrele, K. Olaiya, and B. Sanusi, "Development of a Dynamic Programming Model for Optimizing Production Planning," Dev. A Dyn. Program. Model Optim. Prod. Plan., vol. 2, no. 3, pp. 12-17, 2014.

7. M. Mohammadi and M. Tap, "A Mixed Integer Programming Model Formulation for Solving the Lot-Sizing Problem," Int. J. Comput. Sci. Issues, vol. 9, no. 2, pp. 1694-1703, 2012.

8. H. M. Badri, N. K. Khamis, and M. J. Ghazali, "Integration of lot sizing and scheduling models to minimize production cost and time in the automotive industry," vol. 1, no. 1, pp. 1-14, 2020.

9. C. Afteni and G. Frumuşanu, "A Review on Optimization of Manufacturing Process Performance," Int. J. Model. Optim., vol. 7, no. 3, pp. 139-144, 2017, doi: 10.7763/ijmo.2017.v7.573.

10. F. Ejaz, R. Hashaikeh, A. Diabat, and N. Hilal, "Mathematical and optimization modelling in desalination : State-of-theart and future direction," Desalination, vol. 469, no. July, p. 114092, 2019, doi: 10.1016/j.desal.2019.114092.

11. S. A. Elsheikhi, Mathematical Modeling and Optimization of Injection Molding of Plastics, no. 1. Elsevier Ltd., 2017.

12. A. R. Yusoff, M. R. Z. Mohamed Suffian, and M. Y. Taib, "Literature Review of Optimization Technique for Chatter Suppression in Machining," J. Mech. Eng. Sci., vol. 1, no. December 2011, pp. 47-61, 2011, doi: 10.15282/jmes.1.2011.5.0005.

13. R. T. Marler and J. S. Arora, "Survey of multi-objective optimization methods for engineering," Struct. Multidiscip. Optim., vol. 26, no. 6, pp. 369-395, 2004, doi: 10.1007/s00158-003-0368-6.

14. A. Konak, D. W. Coit, and A. E. Smith, "Multi-objective optimization using genetic algorithms: A tutorial," Reliab. Eng. Syst. Saf., vol. 91, no. 9, pp. 992-1007, 2006, doi: 10.1016/j.ress.2005.11.018.

15. M. A. Ramos, M. Boix, L. Montastruc, and S. Domenech, "Multiobjective optimization using goal programming for industrial water network design,” Ind. Eng. Chem. Res., vol. 53, no. 45, pp. 17722-17735, 2014, doi: 10.1021/ie5025408.

16. N. Brahimi, A. Dolgui, E. Gurevsky, and A. R. Yelles-Chaouche, "A literature review of optimization problems for reconfigurable manufacturing systems,” IFAC-PapersOnLine, vol. 52, no. 13, pp. 433-438, 2019, doi: 10.1016/j.ifacol.2019.11.097.

17. Y. Ye, I. E. Grossmann, J. M. Pinto, and S. Ramaswamy, "Modeling for reliability optimization of system design and maintenance based on Markov chain theory," Comput. Chem. Eng., vol. 124, pp. 381-404, 2019, doi: 10.1016/j.compchemeng.2019.02.016.

18. E. I. Gingu and M. Zapciu, "Markov chains and decomposition method used for synchronizing the manufacturing production rate with real market demand," UPB Sci. Bull. Ser. D Mech. Eng., vol. 79, no. 1, pp. 163-174, 2017.

19. M. Bravo and I. Gonzalez, “Applying stochastic goal programming: A case study on water use planning," Eur. J. Oper. Res., vol. 196, no. 3, pp. 1123-1129, 2009, doi: 10.1016/j.ejor.2008.04.034.

20. E. Ballestero, "Using stochastic goal programming: Some applications to management and a case of industrial production," 


\section{International Journal of Current Science Research and Review}

ISSN: 2581-8341

Volume 05 Issue 02 February 2022

DOI: 10.47191/ijesrr/V5-i2-02, Impact Factor: 5.825

INFOR, vol. 43, no. 2, pp. 63-77, 2005, doi: 10.1080/03155986.2005.11732717.

21. B. Aouni, F. Ben Abdelaziz, and D. La Torre, "The stochastic goal programming model: Theory and applications," $J$. Multi-Criteria Decis. Anal., vol. 19, no. 5-6, pp. 185-200, 2012, doi: 10.1002/mcda.1466.

22. F. Salas-Molina, J. A. Rodriguez-Aguilar, and D. Pla-Santamaria, "A stochastic goal programming model to derive stable cash management policies," J. Glob. Optim., vol. 76, no. 2, pp. 333-346, 2020, doi: 10.1007/s10898-019-00770-5.

23. W. C. Kim, D. G. Kwon, Y. Lee, J. H. Kim, and C. Lin, "Personalized goal-based investing via multi-stage stochastic goal programming," Quant. Financ., vol. 20, no. 3, pp. 515-526, 2020, doi: 10.1080/14697688.2019.1662079.

24. R. Jayaraman, C. Colapinto, D. Liuzzi, and D. La Torre, "Planning sustainable development through a scenario-based stochastic goal programming model," Oper. Res., vol. 17, no. 3, pp. 789-805, 2017, doi: 10.1007/s12351-016-0239-8.

25. K. Eyvindson and A. Kangas, "Stochastic goal programming in forest planning," Can. J. For. Res., vol. 44, no. 10, pp. 1274-1280, 2014, doi: 10.1139/cjfr-2014-0170.

26. M. Kazemi Zanjani, D. Ait-Kadi, and M. Nourelfath, "Robust production planning in a manufacturing environment with random yield: A case in sawmill production planning,” Eur. J. Oper. Res., vol. 201, no. 3, pp. 882-891, 2010, doi: 10.1016/j.ejor.2009.03.041.

27. O. Masmoudi, A. Yalaoui, Y. Ouazene, and H. Chehade, "Lot-sizing in a multi-stage flow line production system with energy consideration,” Int. J. Prod. Res., vol. 55, no. 6, pp. 1640-1663, 2017, doi: 10.1080/00207543.2016.1206670.

28. W. Florim, P. Dias, A. S. Santos, L. R. Varela, A. M. Madureira, and G. D. Putnik, “Analysis of lot-sizing methods' suitability for different manufacturing application scenarios oriented to MRP and JIT/Kanban environments," Brazilian J. Oper. Prod. Manag., vol. 16, no. 4, pp. 638-649, 2019, doi: 10.14488/bjopm.2019.v16.n4.a9.

29. T. Sastri, B. Feiring, and P. Mongkolwana, "Markov chain approach to failure cost estimation in batch manufacturing," Qual. Eng., vol. 13, no. 1, pp. 43-49, 2001, doi: 10.1080/08982110108918623.

30. R. Chatys, "Application of the Markov Chain Theory in Estimating the Strength of Fiber-Layered Composite Structures with Regard to Manufacturing Aspects," Adv. Sci. Technol. Res. J., vol. 14, no. 4, pp. 148-155, 2020, doi: $10.12913 / 22998624 / 126972$.

31. C. T. Papadopoulos, J. Li, and M. E. J. O. Kelly, “A classification and review of timed Markov models of manufacturing systems," Comput. Ind. Eng., vol. 128, no. November 2018, pp. 219-244, 2019, doi: 10.1016/j.cie.2018.12.019.

32. S. Otieno, E. O. Otumba, and R. N. Nyabwanga, "Aplication of markov chain to model and forecast stock market trend: A study of Safaricom shares in Nairobi securities excgange, Kenya," Int. J. Curr. Res., vol. 7, no. 4, pp. 14712-14721, 2015.

33. B. Aouni and D. La Torre, "A generalized stochastic goal programming model," Appl. Math. Comput., vol. 215, no. 12, pp. 4347-4357, 2010, doi: 10.1016/j.amc.2009.12.065.

34. J. Li, L. He, H. Lu, and X. Fan, "Stochastic goal programming based groundwater remediation management under humanhealth-risk uncertainty,” J. Hazard. Mater., vol. 279, pp. 257-267, 2014, doi: 10.1016/j.jhazmat.2014.06.082.

35. T. Language and T. Computing, "MATLAB The Language of Technical Computing," Components, vol. 3, no. 7, p. 750, 2004, doi: 10.1007/s10766-008-0082-5.

36. A. Science, "HPFBU Introduction to MatLab," 2014.

Cite this Article: Maureen Nalubowa S., Paul Kizito Mubiru, Jerry Ochola, Saul Namango (2022). Multi-Objective Optimization of Manufacturing Lot Size Under Stochastic Demand. International Journal of Current Science Research and Review, 5(2), 306-318 